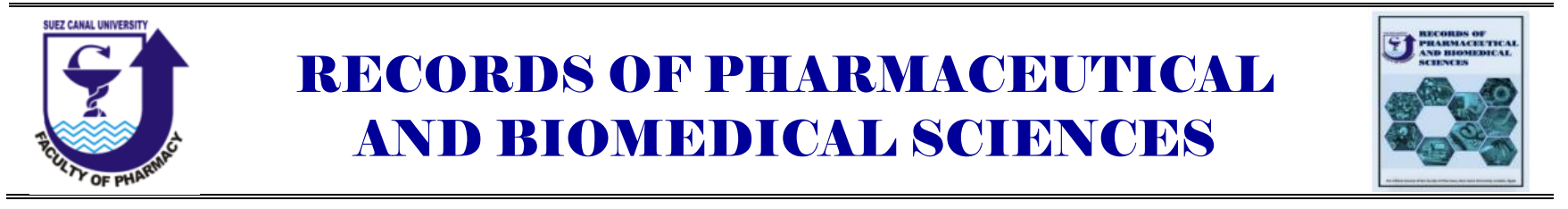

\title{
Insight in Some Herbals and Herbal Combinations Used by Saudi Females for Improving Fertility and Enhance Pregnancy
}

\author{
Hala H. Zaatout* \\ Department of Pharmacognosy, Faculty of Pharmacy, Alexandria University, Alexandria 21521, Egypt
}

Received on: 21.02. 2019

Revised on: 01. 03. 2019

Accepted on: 09. 03. 2019

Correspondence Author:

Tel:+ 201001842369

E-mail address:

halamsan@hotmail.com, hala.zatout@alexu.edu.eg

\begin{abstract}
Delayed pregnancy is one of the main problems that may threaten the relationship between married people especially in Arab region. Finding a cure for this problem started after a period of time after the marriage without pregnancy. In some cases, herbs from tradition can be used in order to promote fertility and stimulate pregnancy. There are limited data for herbal remedies used by women in KSA that is concerned with pregnancy. This study was aimed at determining the prevalence rate and kind of herbal remedies used among female culture. First, a pre-structured questionnaire was administered among 430 Saudi females which revealed that $25 \%$ of them have used traditional herbals as a tool for enhancing the fertility. Then asking apothecary and dealers in that field, collecting samples and data were recorded. Finally, investigating the data collected in attempts to evaluate the efficacy and safety of such herbs and herbal mixtures. Rose of Jericho, Juniper, Myrrh, Black cumin and garden cress were the most commonly used remedies recorded from respondents. This study focuses on possible warning and precaution. Recommendations were suggested to control any hazards.
\end{abstract}

Keywords: Herbals, Saudi, Females, Fertility, Pregnancy.

\section{Introduction:}

Herbs and herbal remedies have played an important role in maintaining health, well-being and disease management for centuries. Traditional medicine uses herbs in the treatment of different ailments. The global demand for herbal medicine has increased significantly in recent years (Ifeoma and Oluwakanyinsol, 2013). Traditional medicine is widely used in the Arabian Peninsula and it has been used to treat conditions of particular interest to women, such as premenstrual syndrome (PMS) and menopausal symptoms (Hardy, 2000). People who are likely to be at risk from adverse effects of herbal medicines include fetus, pregnant women and lactating mothers. While pregnant women and their health care providers are increasingly aware that they should avoid unnecessary exposures during pregnancy, a paradoxical increase in the quantity of self-administered herbal and over the counter(OTC) medication have been reported (Kacew, 1999; Buitendijk and Braken, 1991). The usage of herbal remedies among pregnant women raises particular concerns of safety. These safety concerns have been attributed to the herbal ingredients themselves (Tamuno et al., 2010; Rados, 2004). Herbal remedies are often promoted as natural and safe. These claims attract pregnant women who are often concerned about their unborn child's well-being (Nordeng and Havnen 2005). The traditional use of ethnomedical plants in the KSA represents a 


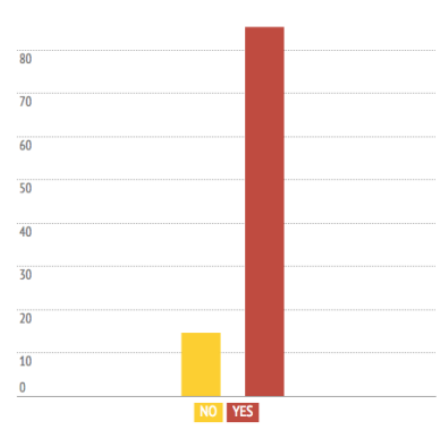

(a)

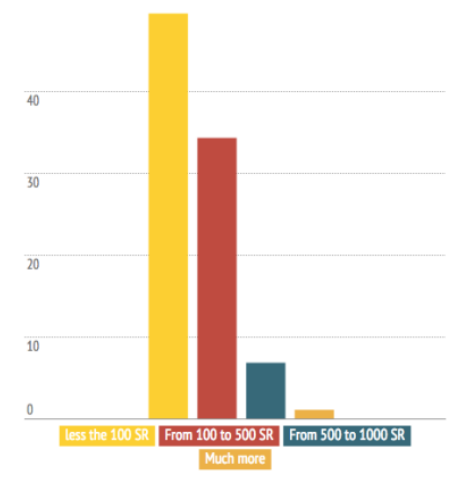

(b)

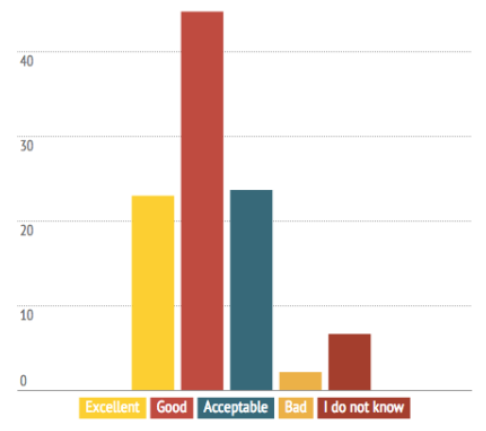

(c)

Figure 1: (a) The usge of herbal remedies after labor, (b) Money spent on these herbal remedies, (c) Satisfaction of using these herbals remedies.

strong interconnection among traditional healing practices characterized by specific cultures. Aati reviews a collection of medicinal plants in KSA used in ethnomedicine (Aati et al. 2019).

\section{Experimental:}

The study was started with the online questionnaire that was created using 'Monkey Survey' application. Social media were used to publish the questionnaire and to collect the data. The responses were expressed as percentages of the total population enrolled in the questionnaire. The variables tested in the study were presented with descriptive statistics. Pearson's second coefficient of skewness is used to measure the asymmetry of a data set. For this quantity, we subtract the mode from the median, multiply this number by three and then divide by the standard deviation. The normality of the data distribution was tested for skewness. Normal distribution was checked using column statistics (modified Kolmogorov-Smirnov). The analysis was performed using GraphPad Prism, software release 3.02. Probability levels less than 0.05 were considered significant (El-Gowelli et al. 2017). Herbal mixtures that are used by Saudi women, in North region, as a treatment for difficulty of pregnancy were recorded. Some apothecaries and traditional healers were asked about these herbals and some samples were collected. Additional herbals were provided by the questionnaire participants' answers.

\section{Results \& Discussion:}

The online questionnaire collected the response of 430 females. Eighty percent of them were around the ages $20-40$. It was found that $25 \%$ of them have used traditional herbals as a tool for enhancing the fertility. $32 \%$ of them have asked for a Doctor's consultation before using these traditional herbals. After labor, $85 \%$ of the sample used the traditional herbals (Figure 1-a), 47\% of them prefer to ask the healer about the components of these mixtures. The majority of the responded females spent not more than a 100 SR for these herbals, while $7 \%$ spent more than 500 SR (Figure 1-b). Data is significantly skewed to the left toward lower money spent. The overall Satisfaction of using these treatments was (Good) with a percentage $44 \%$ (Figure 1-c). Data is significantly skewed to the left toward good satisfaction. The collected herbal mixtures were investigated by photographing and asking apothecaries and Saudi females for their sources, mode of administration, and the expected effects of using these herbs. The main herbal mixtures were recorded.

Herbal Suppository (Figure 2-a) were supplied from a traditional healer and was prescribed to be taken during the first three days of the menstrual cycle for three months while keeping the body worm by using heavy clothes. The expected effect of these suppositories was to clean the uterus and facilitating pregnancy. Almtbokha (Figure 2-b) is a herbal mixture that was supplied from a traditional healer. It was prescribed to be taken during the menstrual cycle by mixing the herbals with warm water and sugar. The expected effect of that mixture was to clean the uterus and helping the woman to be pregnant. Almardoud (Figure 2-c) is a herbal mixture that was supplied from an apothecary. It was prescribed to be eaten with a cup of water or mix it with milk on the $10^{\text {th }}$ day after labor. The expected effect of that mixture was to 
clean the uterus and helping the woman to be pregnant again. Powder mixture I indicated for polycystic ovary (Figure 2-d) was supplied from an apothecary. It was prescribed to be taken as one teaspoonful in warm milk on the second day of the menstrual cycle. The expected effect is to treat the polycystic ovary. Air cpu (Figure 2-e) is a herbal mixture that was supplied from an apothecary.
It was prescribed to be exposed to the smokes of burning the herbals. The expected effect is to treat any inflammation that occurs after birth. Powder mixture II indicated to strengthen the ovaries (Figure 2-f) was supplied from a traditional healer. It is composed of two powders-mixtures; The first one is to be used on the first three days of menstrual cycle.

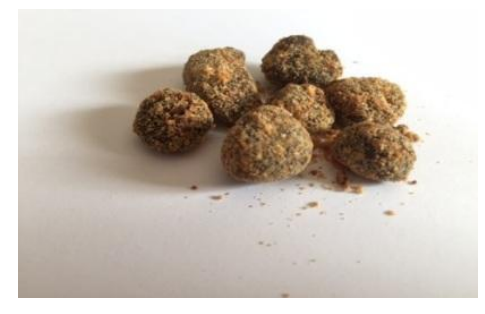

(a)

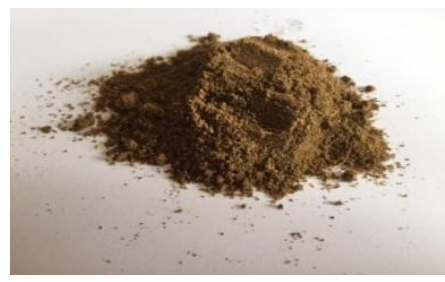

(d)

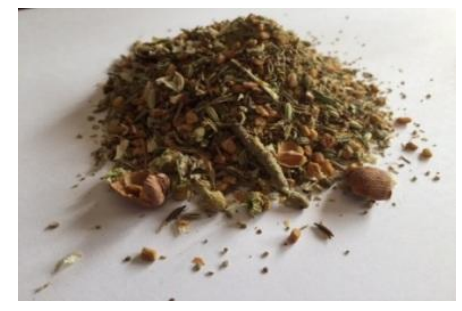

(b)

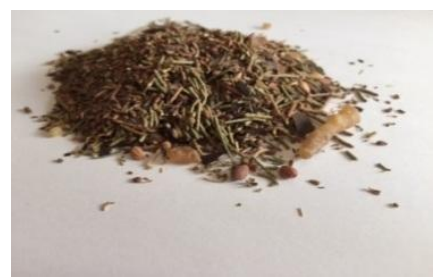

(e)

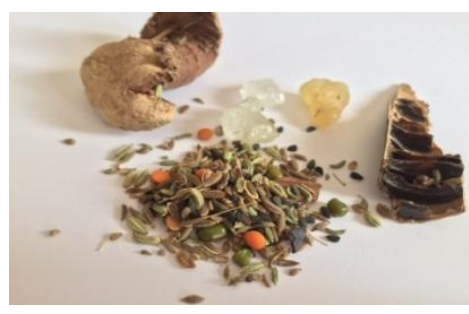

(c)

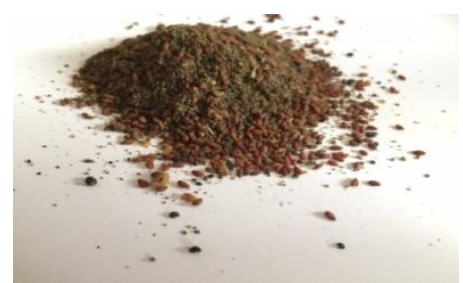

(f)

Figure 2: (a) Herbal suppositories, (b) Almatbokha, (c) Almardoud, (d) Powder mixture I, (e) Air cpu,

\section{(f) Powder mixture II}

The second mixture is to be used from $4^{\text {th }}$ day until the end on the menstrual cycle. The expected effect is to clean the uterus by removing blood clumps that may hinder pregnancy occurrence. Also, it is claimed to strengthen the ovaries. Herbal tablets for pregnant are oral herbal tablets that were supplied from a traditional healer. They were prescribed to be taken twice a day for one week, then one tablet daily until getting pregnant. They were prescribed for both male and female. The expected effect is to induce pregnancy. They were supposed to increase the fertility by increasing the secretion of semen fluid in male and increasing ovulation in female.

The above mentioned data was based on the traditional healers and apothecaries' knowledge and experiences. Some herbals were mentioned in the questionnaire comments that the participants tried for pregnancy, or postnatal. These herbals were recorded as the following: Rose of Jericho, Juniper, Myrrh, Black cumin and Garden cress.

Rose of Jericho is also known as Chastle tree; Vitex agnus-castu. It is used in traditional medicine in cases of dystocia. The plant decoction is taken to facilitate labor. Boiled seeds are claimed to be used with cumin seeds to stop the postpartum bleeding while soaked seeds are used to increase the menstruation (Al-Qahtani, 2008). The drug is dopaminergic and FSH-suppressive; it inhibits lactation. In addition, the drug represses the release of prolactin and improves the symptoms of PMS. Its preparations are used to treat irregularities of the menstrual cycle, premenstrual complaints, menstrual disturbances caused by corpus luteum insufficiency, insufficient milk production and mastodynia. Additional uses include the treatment of impotency, spermatorrhea, prostatitis, swelling of the testes, sexual neurasthenia, sterility, amenorrhea, uterine pain, and swelling of the ovaries. The drug is contraindicated in pregnancy and in nursing mothers (Brendler et al., 2000).

Juniper; Juniperus communis is used to induce menstruation. It is good for tightening the uterus. In folk medicine, it is used internally to regulate menstruation and to relieve menstrual pain. Juniper is contraindicated during pregnancy (Brendler et al., 2000).

Myrrh is sold as Myrrh Commiphora. It is considered as a stimulant for the uterus and it 
increases blood flow. It can be used for the treatment of dysmenorrhea. Also, it is used for amenorrhea and abdominal tumors. Myrrh should not be used during pregnancy because it activates the uterus and can cause abortion (Brendler et al., 2000). Black cumin; Nigella sativa seeds were indicated to be used by mixing half teaspoon with two-thirds cup of water and to be orally taken daily. The expected effect is the treatment of dysmenorrhea. The use of black seed during pregnancy should be prohibited because its oil causes abortion (Al-Qahtani, 2008).

Lepidium sativum is commonly known as Common cress, Garden cress. Lepidium sativum seed extract showed laxative activities (Hassan et al., 2011). In South Asia, it is used in traditional medicine to treat asthma, bronchitis, and cough. It is considered useful as abortifacient, antibacterial, aphrodisiac, diuretic, expectorant, gastrointestinal stimulant, gastroprotective and stomachic (Baquar, 1989 and Al-Qahtani, 2008). Lepidium sativum seeds should not be used during pregnancy.

\section{Conclusion and recommendation:}

The use of herbal remedies in Arab cultures is widely spread due to the false concepts and misunderstanding. Some herbs may be abortifacient or may cause severe consequences on both mother and her fetus. More information is required for proper identification of these herbals.

The use of herbals should be regulated according to public health organizations' rules. Academic and specialized people should show their responsibilities toward the community through elevating the awareness about safe and effective use of herbal remedies. Public health authorities should prevent unspecialized people activity to prevent the misuse of people ignorance.

\section{Conflict of interest}

The authors have no conflict of interest to declare.

\section{Ethical approval}

Not applicable.

\section{Source of funding}

The study did not receive any specific grant from funding agencies in the public, or commercial sectors.

\section{Acknowledgements}

The author like to acknowledge saudi colleagues for their help in collecting the herbal mixture samples.

\section{References}

Aati, H., El-Gamal, A., Shaheen, H. and Kayser, O. 2019. Traditional use of ethnomedicinal native plants in the Kingdom of Saudi Arabia. Journal of Ethnobiology and Ethnomedicine, 15:2

Al-Qahtani, J. (2008). Jaber Encyclopedia of Herbal Medicine. $2^{\text {nd }}$ Edition, Al-Obekan Library, Riyadh, Saudi Arabia.

Baquar, S. R. (1989). Medicinal and Poisonous Plants of Pakistan, Printas, Karachi, Pakistan.

Brendler, T., Wyble, C., Hamid, M., Nathan, J., Potter, J. C., Rodgers, K. and Phayre, A. M. (2000). PDR for Herbal medicines. $4^{\text {th }}$ Edition, Thomson Healthcare.

Buitendijk, S. and Braken, M. B. 1991. Medication in Early Pregnancy; Prevalence of Use and Relationship to Maternal Characteristics. Amercian Journal Obstetrics \& Gynecology; 165: 33 - 40.

El-Gowelli HM, Ibrahim KS, El-Yazbi AF, El-Mas MM. (2017). Role of NADPHox/Rho-kinase signaling in the cyclosporine-NSAIDs interactions on blood pressure and baroreflexes in female rats. Life Sci. 15;185:15-22.

Hardy, M. L. 2000. Herbs of special interest to women. Journal of the American Pharmaceutical Association, 40(2):234-242.

Hassan, M., Alkharfy, K. M., and Gilani, A. 2011. Prokinetic and laxative activities of Lepidium sativum seed extract with species and tissue selective gut stimulatory actions. Journal of ethnopharmacology, 134(3), $\quad$ 878-883. https://doi.org/10.1016/j.jep.2011.01.047.

Ifeoma, O. and Oluwakanyinsol, S. (2013). Screening of Herbal Medicines for Potential Toxicities. New Insights into Toxicity and Drug Testing (Vol. i, p. 13). InTech. https://doi.org/10.5772/54493

Kacew S. 1999. Effects of Over the Counter Drugs on the Unborn Child: What is Known and How Should this Influence Prescribing? Paediatric Drugs, 1: $75-80$. 
Nordeng, H. and Havnen, G. C. 2005. Impact of socio-demographic factors, knowledge and attitude on the use of herbal drugs in pregnancy, Acta Obstetricia et Gynecologica Scandinavica, 84(1), 26-33.

Rados C. (2004). Ephedra Ban: no shortage of Reasons. FDA Consum; 38: 6-7.
Tamuno, I., Omole-Ohonsi, A. and Fadare, J. 2010. Use of Herbal Medicine Among Pregnant Women Attending a Tertiary Hospital in Northern Nigeria. The Internet Journal of Gynecology and Obstetrics, 15 (2), 1-8. 